\title{
PREDICTIVE WATERSHED FOR IMAGE SEQUENCES SEGMENTATION
}

\author{
Shao-Yi Chien*, Yu-Wen Huang, Shyh-Yih Ma, and Liang-Gee Chen \\ DSP/IC Design Lab \\ Department of Electrical Engineering, National Taiwan University \\ 1, Sec. 4, Roosevelt Rd., Taipei, Taiwan
}

\begin{abstract}
Watershed transform is a key operator in video segmentation algorithms. In this paper, a new fast watershed algorithm named P. Watershed for image sequences segmentation is proposed. By utilizing the temporal coherence property of video signal, this algorithm updates watersheds instead of searching watersheds in whole image. The watershed process can be accelerated and the segmentation results are almost the same as those of conventional algorithms. Moreover, an intra-inter watershed scheme (IP-Watershed) is also proposed to further improve the results. Experimental results show this algorithm can save 50\% computation without degrading the segmentation results.
\end{abstract}

\section{INTRODUCTION}

Watershed transform, which can separate an image into many homogeneous non-overlapped close regions, has been widely applied in image segmentation algorithms. It is also applied on image sequences as a core operator of video segmentation [1], which is a key technique in MPEG-4 content-based encoding systems [2]. Video segmentation algorithms with watershed transform $[3,4,5$, 6] are seen as mainstream since they can generate object masks with accurate boundaries.

A lot of watershed algorithms have been proposed $[7,8,9]$. Vincent and Soille proposed a watershed algorithm based on immersion simulations [7]. With sorting before flooding process and with priority queue, this algorithm is dramatically faster than any former ones. Meyer's algorithm is also based on immersion simulations [8]. Ordered queue is used in this algorithm, whose concept is similar to Vincent's algorithm. Moga, Cramariuc, and Gabbouj proposed a watershed algorithm suitable for parallel implementation [9]. However, it is complex and a powerful platform is needed, which is impractical for general cases.

Although Vincent's algorithm provides fast computation; however, for real-time video segmentation, it is still not fast enough. Besides, video segmentation is different from image segmentation. The temporal information is important in video signal processing. Applying image signal processing algorithms on each frame for video signal will lead to redundant computation.

A predictive watershed algorithm (P-Watershed) for image sequences segmentation is proposed in this paper. It is based on the fact that watersheds are highly related to original frame data. It "updates" watersheds instead of recalculating watershed transform frame by frame. The watershed process can be accelerated, and the results are almost the same as those of conventional watershed

\footnotetext{
* This work is supported by SiS Education Foundation.
}

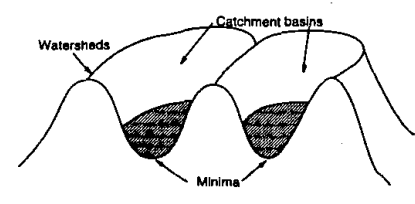

Fig. 1. Illustration of watersheds.

algorithms. Furthermore, the segmentation results can be further improved with intra-inter watershed scheme (IP-Watershed).

The concepts of watershed and Vincent's algorithm will be introduced in Sec. 2. After that, the proposed algorithm and the simulation results will be shown in Sec. 3 and Sec. 4. Finally, Sec. 5 gives a short conclusion.

\section{WATERSHED}

\subsection{Morphological waterhsed}

Watershed transform is originally developed in the field of topography, and it is found to be useful in digital image processing. The first step of morphological watershed is morphological gradient. The gradient level of an image is then seen as altitude level to form topology surface. When water is flooded into this surface, it will flow to the lower parts of this surface because of gravity. As shown in Fig. 1, water will flow to minima of each catchment basins. If we label water in each catchment basin a special label and keep flooding water, water from different catchment basins will interflow at watersheds. Watersheds, which correspond to high gradient level parts of an image, are then detected, and they can be used to separate an image into many homogeneous close regions.

The flooding process is often done with two mechanisms: immersion or rainfall. The immersion simulation is more popular and is described below. First, we pierce holes at minimun of each catchment basin, and water is flooded from these holes to form small lakes. When water from different catchment basins is going to interflow, a "dam" is build. After all the surface is flooded, these dams correspond to the watersheds of this image.

\subsection{Vincent's algorithm}

Vincent's algorithm [7] is the most famous one and has been thought as the fastest watershed algorithm [10]. This algorithm can be briefly illustrated in Fig. 2 and following steps.

Step 1: All the pixels are first sorted according to their gradient level. Pixels with lower gradient level will be manipulated first. 


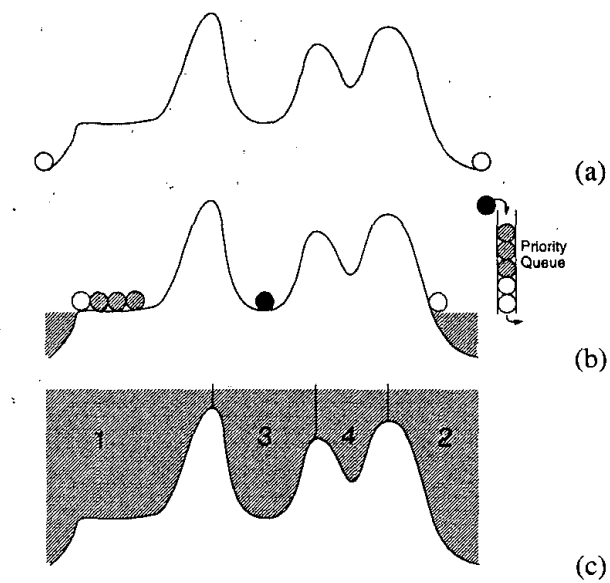

Fig. 2. Illustration of.Vincent's watershed process.

Step 2: As shown in Fig. 2(a), pixels with lowest gradient level of an image are first found, and pixels belong to each connected region are given a special label.

Step 3: Pixels are then processed from lower gradient level to higher level. In each level, pixels with labeled neighbors are first added to the priority queue as shown in Fig. 2(b). The label of the pixel at the beginning of the queue is determined with its labeled neighbors, and the pixel is then removed from the queue. After all pixels with labeled neighbors are processed, pixels without labeled neighbors are found and given a new label, as the black ball in Fig. 2(b).

Step 4: After all pixels are labeled, the boundaries between regions with different labels are the watersheds of this image. In Fig. $2(c)$, the surface is divided into four regions, and three watersheds are detected.

\section{PROPOSED WATERHSED ALGORITHM}

For image sequences segmentation, Vincent's algorithm can be further accelerated by consideration of temporal coherence of sequences. The core concepts of the proposed algorithm are: (a) watersheds are highly related to original image sequences; (b) temporal coherence of image sequences leads to redundant computation when watershed transform is applied on whole image frame by frame, that is, the process can be accelerated with "updating" scheme; (c) the computational intensity of watershed algorithm is proportional to processed pixel number, that is, reducing pixel number can accelerate the process; (d) since watershed is a global operation, the algorithm should be modified in the updating area to keep the globalization. With these concepts, a new algorithm is proposed as follows.

\subsection{Predictive watershed}

The block diagram of proposed watershed algorithm is shown in Fig. 3. The first frame of an image sequence is processed with conventional Vincent's algorithm, and the region label and gradient information are kept. From second frame, the proposed watershed algorithm named predictive watershed (P-Watershed) takes over, and the conventional watershed process is turned off.
Current frame and corresponding frame are then inputted into block-based change detection to form updating area mask (UAM), and corresponding frame is then updated with current frame and $U A M$. The operation can be formulated as following equations: for each block,

$$
\begin{gathered}
S A D=\sum_{x, y \in \text { each block }} \mid \text { CFrame }_{x, y}-\text { CorFrame }_{x, y} \mid, \\
U A M=\left\{\begin{array}{ll}
1 & \text { if } S A D \geq T h \\
0 & \text { else }
\end{array},\right. \\
\text { CorFrame }_{x, y}=\left\{\begin{array}{ll}
\text { CFrame } & \text { if } U A M=1 \\
\text { CorFrame } & \text { else }
\end{array},\right.
\end{gathered}
$$

where CFrame is current frame, and CorFrame is corresponding frame. Corresponding frame stores the original frame data whom current watersheds corresponds to.

After $U A M$ is generated, it can indicate changing parts of the image, and the corresponding watersheds should be updated. Next, morphological gradient and sorting are applied only on pixels in updating area $(U A)$, which is indicated by $U A M$. The watershed process is then applied only in $U A$. The whole process is shown in Fig. 4 and following steps.

Step 1: Fig. 4(a) shows the region label and gradient value of previous frame, and updating area is marked.

Step 2: As shown in Fig. 4(b), region labels and gradient values in $U A$ are removed, and morphological gradient operation is then applied in $U A$ to generate gradient values of current frame.

Step 3: Pixels in UA are sorted according to gradient level.

Step 4: Pixels in $U A$ are then processed from lower gradient level to higher level. In each level, the process is the same as the step 3 of Vincent's algorithm. Note that neighbors outside $U A$ are also taken into consideration, as shown in Fig. 4(c). After all pixels with labeled neighbors are processed, pixels without labeled neighbors are found and given a new label, as the white ball in Fig. 4(c).

Step 5: After all pixels are labeled, the boundaries between regions with different labels are the watersheds of this image. In Fig. 4(d), two watersheds are detected.

An example of P-Watershed is shown in Fig. 5. In Fig. 5(a) previous frame and its watersheds are shown. The UAM is shown in Fig. 5(b), and the watersheds in $U A$ are then generated as shown in Fig. 5(c). Finally, in Fig. 5(d), the watersheds of current frame are formed by updating watersheds in $U A$.

Only part of pixels are processed, hence the proposed algorithm is faster than conventional watershed algorithm. Pixels inside $U A$ are processed with conventional algorithm, and region label and gradient information outside $U A$ are also taken into consideration; therefore, the globalization property is also kept with this scheme.

\subsection{Hybrid scheme}

Although the segmentation results are almost the same as conventional watershed algorithms, the proposed algorithm may introduce error when the block-based change detection is not sensitive enough or when the gradient value across the boundaries of $U A$ is discontinuous. The error will be propagated in the proposed predictive watershed algorithm. The error propagation can be interrupted by inserting watersheds generated with conventional algorithms. These watersheds do not need information in previous frame so it is named as "intra watershed" (I-Watershed). The I- 


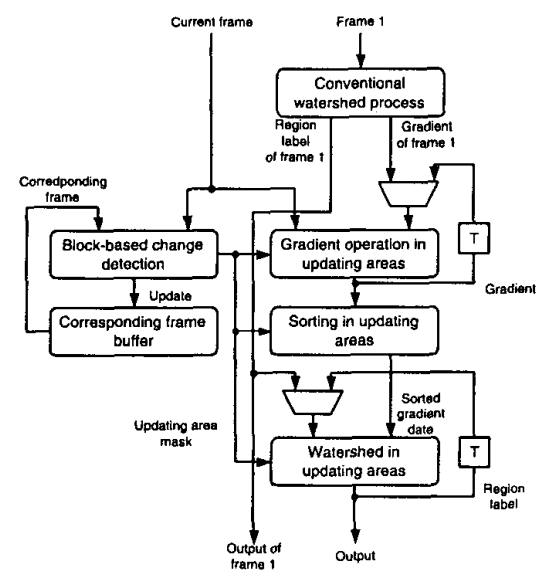

Fig. 3. Block diagram of proposed watershed algorithm.
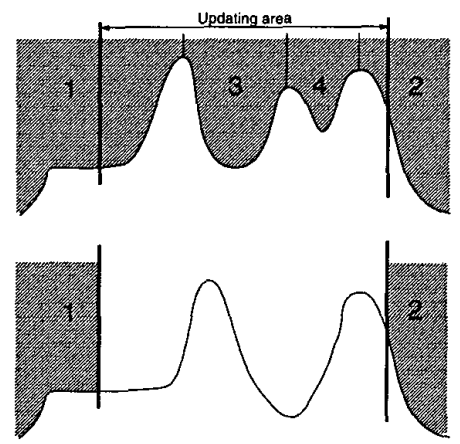

(a)

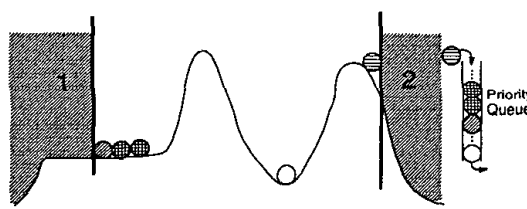

(b)

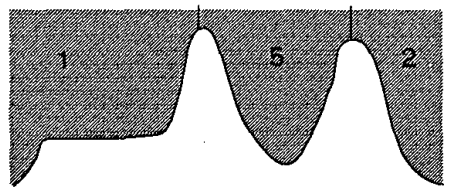

(c)

(d)

Fig. 4. Illustration of proposed watershed process.

Watershed and P-Watershed hybrid scheme, which is named as IP-watershed, can further promote correctness of accelerated watershed process.

\section{SIMULATION RESULTS}

In these experiments, the block size is chosen as $8 \times 8$ since several sequences have been tested to show that it is the optimal choice. Segmentation results of Vincent's algorithm are used as references. The correctness of segmentation results is evaluated with segmented region number, that is, the less difference of region number between experimental results and reference results, the higher cor-

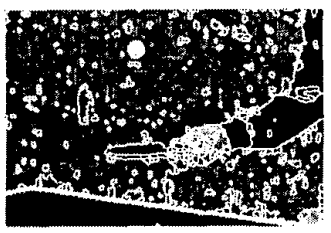

(a)

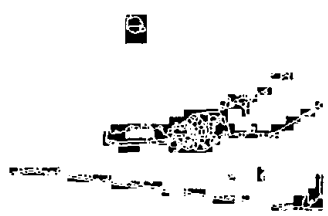

(c)

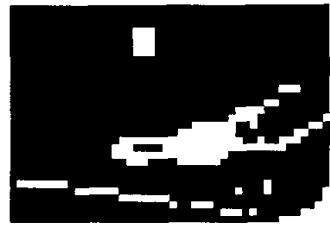

(b)

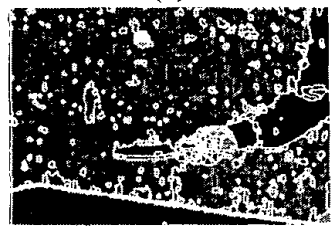

(d)
Fig. 5. Example of proposed watershed algorithm.

Table 1. Run-time comparison between proposed algorithm and Vincent's algorithm.

\begin{tabular}{lllll}
\hline Sequence & Size & $\begin{array}{l}\text { (a) Vincent's } \\
\text { (ms/frame) }\end{array}$ & $\begin{array}{l}\text { (b) Proposed } \\
\text { (ms/frame) }\end{array}$ & $\begin{array}{l}\text { Ratio(\%) } \\
=(b) /(a)\end{array}$ \\
\hline Akiyo & $360 \times 243$ & 202.16 & 107.64 & 53.24 \\
Weather & $360 \times 243$ & 203.89 & 103.15 & 50.59 \\
Hall & $360 \times 240$ & 199.77 & 160.21 & 80.20 \\
Children & $360 \times 240$ & 200.02 & 128.61 & 64.30 \\
$\begin{array}{l}\text { Mother and } \\
\text { daughter }\end{array}$ & $176 \times 144$ & 55.36 & 41.87 & 75.63 \\
\hline
\end{tabular}

rectness. Since regions in $U A$ and regions outside $U A$ are both processed with conventional watershed algorithm, the position of watersheds mush be correct and only over-segment problem may occur, the region number information is enough to evaluate the correctness of watershes. The region number corresponding to different run-time situations are shown in Fig. 6. Run-time can be adjusted by changing the parameter $T h$ and is presented by the ratio to that of conventional watershed algorithm. It shows that when the run-time increases, the similarity between the results of conventional watershed and proposed one also increases. When the run-time is reduced to $61 \%$, the region number is still very similar to the reference results.

The segmentation results of Vincent's algorithm and the proposed one are shown in Fig. 7. Sequence Akiyo, Hall, and Mother and Daughter are tested on a PC with Pentium-III $800 \mathrm{MHz}$ processor. The execution time compared with Vincent's algorithm is shown in Table 1. Only core operations of watershed are recorded, that is, gradient operation, sorting process, and flooding process. Note that the average difference of region number is fixed to $5 \%$ in these experiments. The simulation results show that, with proposed algorithm, 20\% 50\% computation are saved. Besides, the results of conventional and proposed algorithm are quite the same as shown in Fig. 7. Obviously, the proposed algorithm can reduce computational intensity as well as keep the segmentation quality.

The simulation results of IP-Watershed are shown in Fig. 8 and Fig. 9. Note that in these experiments, I-Watershed is inserted every 20 frames. Fig. 8 shows that compared with P-Watershed, IP-Watershed can give results more similar to those of conventional algorithms. The segmentation quality can be improved with IP-Watershed as shown in Fig. 9. 


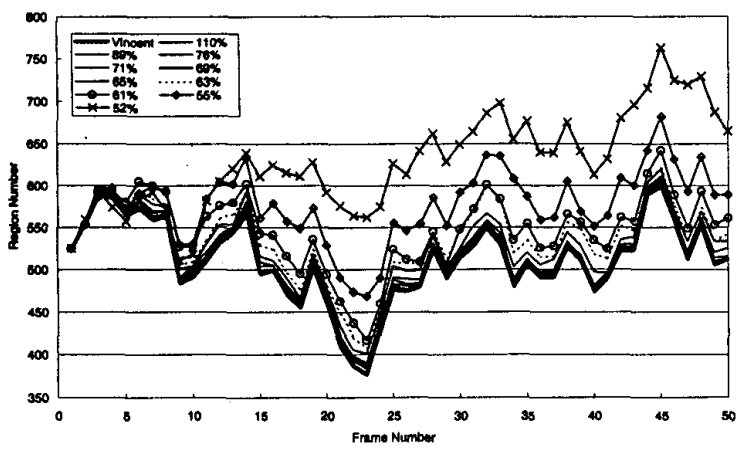

Fig. 6. Region number of different run-time situations.

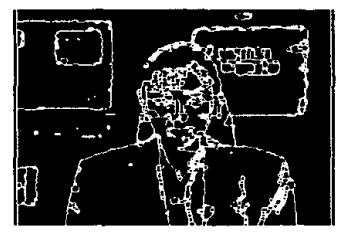

(a)

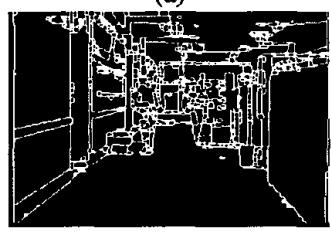

(c)

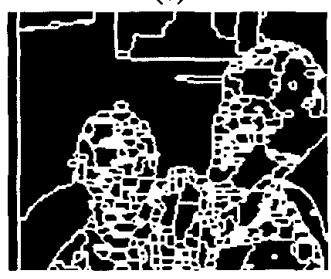

(e)

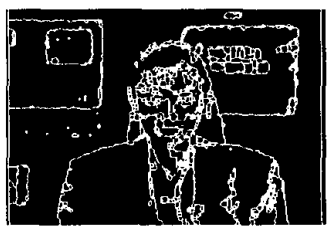

(b)

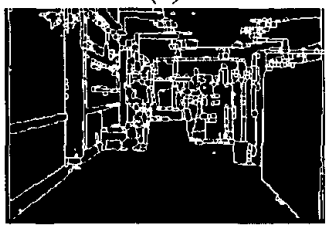

(d)

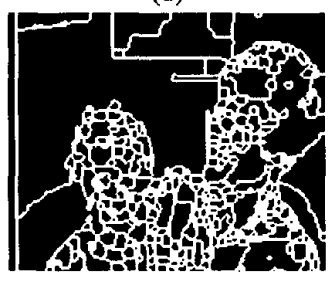

(f)
Fig. 7. (a)(c)(e): watersheds generated with conventional watershed algorithm; (b)(d)(f): watersheds generated with proposed algorithm. The 50th frame of these sequences are shown.

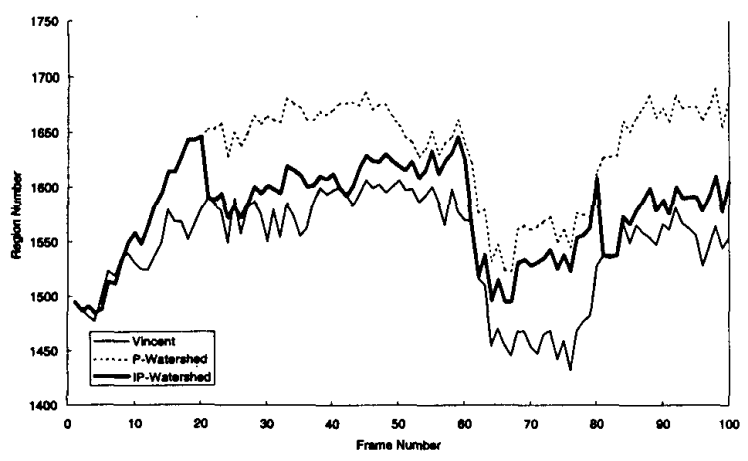

Fig. 8. Simulation results of IP-Watershed (Weather).

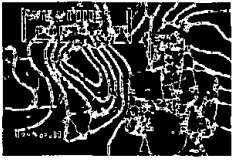

(a)

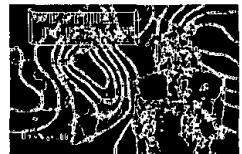

(b)

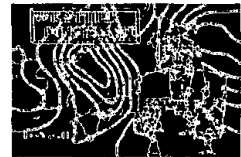

(c)
Fig. 9. Watersheds generated with (a) conventional watershed algorithm, (b) P-Watershed algorithm, and (c) IP-Watershed (insert I-watershed every 20 frames).

\section{CONCLUSION}

A new predictive watershed algorithm named $\mathrm{P}$-Watershed for image sequences segmentation is proposed in this paper. It updates watersheds in changing parts and keeps watersheds in other parts of a frame. The watershed process can be accelerated, and the results are almost the same as conventional watershed algorithms. The segmentation results, moreover, can be further improved with intra-inter watershed scheme, which is named IP-Watershed.

\section{REFERENCES}

[1] Y.-W. Huang, S.-Y. Chien, S.-Y. Ma, and L.-G. Chen, "Analysis of global motion effects on video segmentation," in Proc. of the 2000 Asia Pacific Conference on Multimedia Technology and Applications, 2000, pp. 106-116.

[2] T. Sikora, "The MPEG-4 video standard verification model," IEEE Transactions on Circuits and Systems for Video Technology, vol. 7, no. 1, pp. 19-31, Feb. 1997.

[3] MPEG Video Group, "Annex f: Preprocessing and postprocessing," ISO/IEC JTC I/SC 29/WGII N3056.

[4] D. Wang, "Unsupervised video segmentation based on watersheds and temporal tracking," IEEE Transactions on Circuits and Systems for Video Technology, vol. 8, no. 5, pp. 539-546, Sept. 1998.

[5] Jae Gark Choi, Si-Woong Lee, and Seong-Dae Kim, "Spatiotemporal video segmentation using a joint similarity measure," IEEE Transactions on Circuits and Systems for Video Technology, vol. 7, no. 2, pp. 279-286, Apr. 1997.

[6] D. K. Park, H. S. Yoon, and C. S. Won, "Fast object tracking in digital video," IEEE Transactions on Consumer Electronics, vol. 46, no. 3, pp. 785-790, Aug. 2000.

[7] L. Vincent and P. Soille, "Watersheds in digital spaces: an efficient algorithm based on immersion simulations," IEEE Transactions on Pattern Analysis and Machine Intelligence, vol. 13, no. 6, pp. 583-598, June 1991.

[8] F. Meyer, "Color image segmentation," in International Conference on Image Processing and Its Applications, 1992, pp. 303-306.

[9] A. Moga, B. Cramariuc, and M. Gabbouj, "An efficient watershed segmentation algorithm suitable for parallel implementation," in Proc. of International Conference on Image Processing, 1995, pp. 101-104.

[10] D. Hagyard, M. Razaz, and P. Atkin, "Analysis of watershed algorithms for greyscale images," in Proc. of International Conference on Image Processing, 1996, pp. 41-44. 